\title{
An Approach on the Hydrogen Absorption in Carbon Black after Gamma Irradiation
}

\author{
Antonio Madroñero ${ }^{1}$, Mario Culebras ${ }^{2}$, Andrés Cantarero ${ }^{2}$, Clara M. Gómez ${ }^{2}$, César Mota ${ }^{1}$, \\ José M. Amo' ${ }^{1}$, José I. Robla ${ }^{1}$ \\ ${ }^{1}$ Centro Nacional de Investigaciones Metalúrgicas (CSIC), Madrid, Spain \\ ${ }^{2}$ Instituto de Ciencia de los Materiales, Universidad de Valencia, Valencia, Spain \\ Email: cesarmotacarracedo@gmail.com,mario.culebras@uv.es, andres.cantarero@uv.es, clara.gomez@uv.es, \\ inmac09@gmail.com, amoortega@cenim.csic.es,jrobla@cenim.csic.es
}

Received August 25, 2013; revised September 28, 2013; accepted October 14, 2013

Copyright (C) 2013 Antonio Madroñero et al. This is an open access article distributed under the Creative Commons Attribution License, which permits unrestricted use, distribution, and reproduction in any medium, provided the original work is properly cited.

\begin{abstract}
In this work, different samples of an industrial carbon black are used to study the hydrogen intake from an over pressurized atmosphere and its changes due to alteration of its level of crystallinity produced by $\gamma$-irradiation. The monitoring of the hydrogen adsorption was made by means of thermogravimetric analysis and by measurements of some electrical parameters as the Seebeck coefficient. X-ray diffraction shows that the irradiation diminishes the level of crystalline perfection. These results show interesting possibilities to use carbon black as cheap hydrogen absorbers.
\end{abstract}

Keywords: Carbon Black; Hydrogen Storage; Carbon Semiconductor; Seebeck’s Effect; Gamma Irradiation

\section{Introduction}

As it is well known, hydrogen plays a double role in carbons when the carbons store hydrogen showing electrical parameters figures that correspond to semiconductor materials [1]. As a result, to increase the hydrogen storage capability of carbons is a good path to obtain cheap semiconducting carbons. As the hydrogen intake starts in the surface of the solids, many works were oriented to the improvement of the surface paying attention to the optimization of the hydrogen adsorption. Following this direction, as it is commonly accepted, the amount of defects and the distribution and sizes of them were the main parameters to achieve a good hydrogen adsorption. The linear relationship between the hydrogen uptake and the specific surface area (SSA) is independent of the nature of the carbon material [2]. In the development of carbonaceous absorbers for hydrogen storage there are many studies showing different techniques to activate the carbon's surface, with chemicals [3] and with gas etching [4]. Most of the commercial active carbons available nowadays to store hydrogen correspond to this stage. After that, the possibility was explored to use physical treatments to produce the required defects. Radiation by $\gamma$-rays is a powerful tool to produce defects in the surface and inner defects in carbons [5].
In carbon nanotubes, $\gamma$-irradiation had been more effective than chemical etching to activate carbon surfaces [6]. In the present work, we will study the capability of $\gamma$-rays to increase the semiconducting character of carbon black samples to increase the hydrogen absorption.

\section{Materials}

The material used in the present work was from Black Pearls 1400, manufactured by Cabot ${ }^{\mathrm{TM}}$. According to the manufacturer's information, the specific surface of this material is $560 \mathrm{~m}^{2} / \mathrm{g}$. We have selected carbon black specimens with the best performance. The surface energy of commercial carbons blacks is from $70-200 \mathrm{~m}^{2} / \mathrm{g}$ [7] and the figure of the surface area is a liable identifier of carbons with a good capacity for hydrogen adsorption [8]. The samples were subjected to two successive treatments: hydrogenation and irradiation. The hydrogenation was performed in a pressurize hydrogen atmosphere at 20 bar and room temperature for 180 minutes. In these conditions, a hydrogen adsorption on the carbon black powder took place. The used hydrogen was Ultrapure Plus $\times 50 \mathrm{~S}$ $(99.9992 \%)$, supplied by Carburos Metalicos ${ }^{\mathrm{TM}}$. In the irradiation process, the sample was exposed to a 504-kGy irradiation with ${ }^{60} \mathrm{Co}$ isotopes. The sample exposure to the radioisotopes was carried out by immersion in a wa- 
ter well where the radioisotopes were located. The location was the Nayade facility, existing at the Centre of Energy, Environment and Technology Research (Centro de Investigaciones Energéticas, Medioambientales y Tecnólogicas, CIEMAT), in Madrid, Spain.

For this study, four samples are prepared in order to study hydrogenation and irradiation effects in the samples as summarized in Table $\mathbf{1}$.

\section{Methods}

\subsection{Thermogravimetrical Analysis}

The four samples analysed here were examined by Thermal Gravimetry (TGA), using the equipment DTA/TGA SETARAM Setsys Evolution. The weight of the four samples was around $13 \mathrm{mg}$.

The measurement supposed a heating from room temperature to $1073 \mathrm{~K}$ in an argon atmosphere $(20 \mathrm{ml} / \mathrm{min})$. The heating rate for the four samples was:

1) From room temperature up to $673 \mathrm{~K}$ : the heating rate was $5 \mathrm{~K} / \mathrm{min}$.

2) From $673 \mathrm{~K}$ up to $873 \mathrm{~K}$ : the heating rate was 3 $\mathrm{K} / \mathrm{min}$.

3) From $873 \mathrm{~K}$ to $1073 \mathrm{~K}$ : the rate was $5 \mathrm{~K} / \mathrm{min}$.

The TGA curves were recorded using the software CALISTO v1.0.95.

\subsection{X-Ray Diffraction}

Another technique of examination was X-Ray Diffraction (XRD), performed in a Siemens D5000 diffractometer with Ni-filtered $\mathrm{Cu} \mathrm{K} \mathrm{K}_{\alpha}$ radiation. The X-ray tube was operated at $40 \mathrm{kV}$ and $30 \mathrm{~mA}$. The experimental diffractometers were collected with a step of $0.03^{\circ}(2 \theta)$ and an averaging time of $0.6^{\circ} / \mathrm{min}$. The XRD patterns of the samples were identified with the Joint Committee on Powder Diffraction Standards (JCPDS) files.

\subsection{Electrical Parameters Measurements}

The samples were compacted in thin pellets and four contacts with silver paste were deposited on the surface for electric characterization. In order to measure the electrical conductivity, the Van der Pauw method was used [9]. The electrical conductivity can be obtained solving

Table 1. Treatments in the carbon black samples.

\begin{tabular}{cc}
\hline Sample & Treatment \\
\hline TN-sH & without treatment \\
$\mathrm{TN}-\mathrm{H}$ & Hydrogenation \\
$\mathrm{CN}-\mathrm{sH}$ & Irradiation \\
$\mathrm{CN}-\mathrm{H}$ & Irradiation and hydrogenation \\
\hline
\end{tabular}

the Van der Pauw equation:

$$
\mathrm{e}^{-\pi \cdot d \cdot R_{1} \cdot \sigma}+\mathrm{e}^{-\pi \cdot d \cdot R_{2} \cdot \sigma}=1
$$

To calculate $R_{1}$ and $R_{2}$ four contacts, labelled $A, B, C$ and $D$, were used. $R_{1}$ is obtained as $R_{1}=V_{B D} / I_{A C}$ and $R_{2}=V_{A B} / I_{C D}$, were $V$ and $I$ are the voltage and intensity across the sample, respectively. A Keithley 2400 multimeter was used as a current source.

The Seebeck coefficient is determined as the ratio between the electrical potential, $\Delta V$, and the temperature difference, $\Delta T$, that is:

$$
S=\frac{\Delta V}{\Delta T}
$$

For the temperature control, a "Lakeshore 340 Temperature Controller" was used and for recording the potential data a "Keithley 2750 Data Acquisition Switching System".

\section{Results and Discussion}

Figure 1 shows the results of the TGA. The more relevant feature in sample $\mathrm{CN}-\mathrm{H}$ is that there is an increase in weight, more pronounced at temperatures about 500 $700 \mathrm{~K}$. It is easy to explain it as a process of argon absorption $[10,11]$ that takes place in the surface of the carbonaceous materials. In samples TN-H, CN-sH and TN$\mathrm{sH}$, Ar adsorption at the sample surface was not observed.

On the other hand, in Table 2, the weight loss, a parameter that supplies information about the hydrogen storage I shown. It is simple to see that the irradiated samples stores more hydrogen that the non-irradiated ones. A figure of hydrogen content larger than $10 \% \mathrm{w} / \mathrm{w}$ has interest looking at the possibility of use carbon blacks as cheap hydrogen adsorbers in other fields of the hydrogen economy as in the construction of portable canister with stored hydrogen for the automotive industry.

In Figure 2, referred to non-hydrogenated samples, it

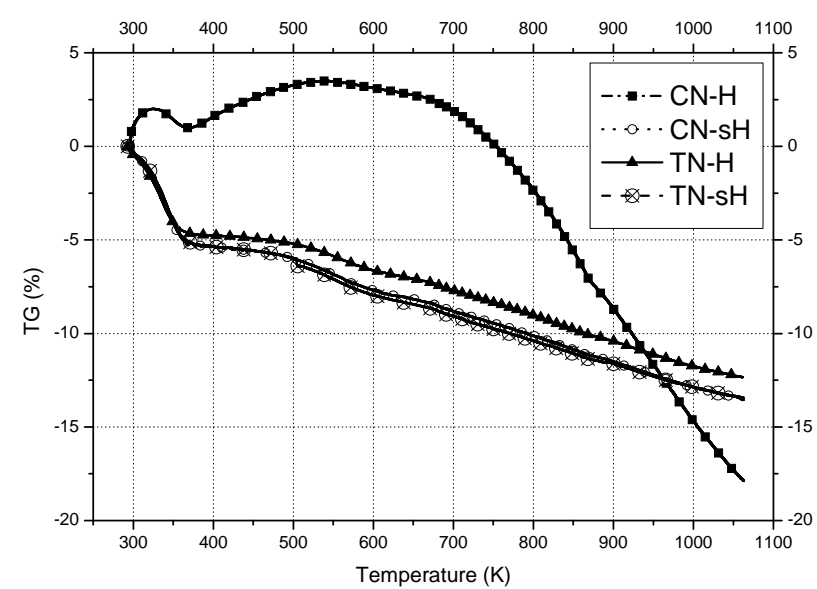

Figure 1. Thermal gravimetric analysis of the samples. 


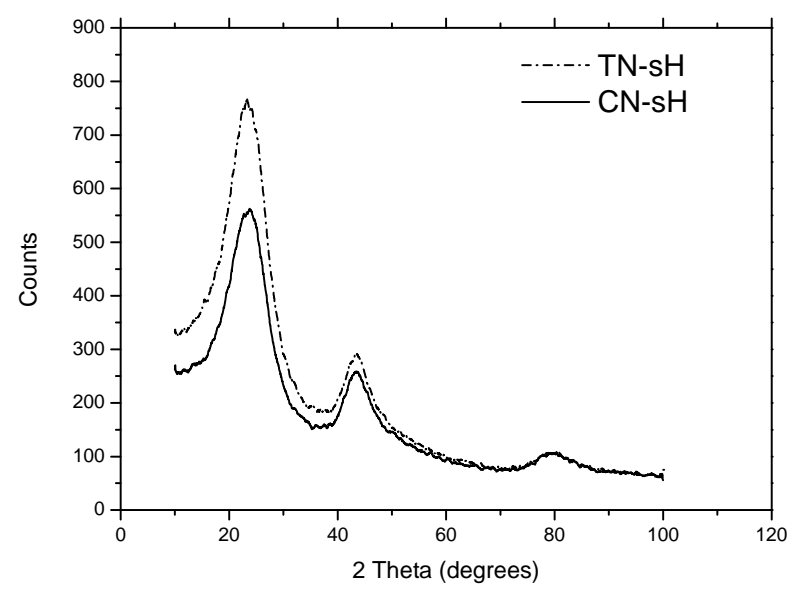

Figure 2. XRD plot of non-hydrogenated samples.

Table 2. Weight loss (in \%) during the TGA showing the variations in the different temperature ranges.

\begin{tabular}{ccccc}
\hline \multirow{2}{*}{ Sample } & $\Delta m_{1}(\%)$ & $\Delta m_{2}(\%)$ & $\Delta m_{3}(\%)$ & $\Sigma \Delta m(\%)$ \\
\cline { 2 - 4 } & $(273-673 \mathrm{~K})$ & $(673-873 \mathrm{~K})$ & $(873-1073 \mathrm{~K})$ & \\
\hline $\mathrm{CN}-\mathrm{H}$ & 1.596 & -9.877 & -10.444 & -18.69 \\
$\mathrm{CN}-\mathrm{sH}$ & -1.184 & -2.826 & -2.329 & -13.0 \\
$\mathrm{TN}-\mathrm{H}$ & -7.167 & -2.839 & -2.219 & -12.11 \\
$\mathrm{TN}-\mathrm{sH}$ & -8.338 & -2.723 & -1.987 & -13.0 \\
\hline
\end{tabular}

is possible to see that the effect of the irradiation is to diminish the crystalline perfection displayed as an impairing of the slenderness of the diffraction peak; the irradiated carbon black becomes more similar to an amorphous sample. The fact that $\gamma$ irradiation increases the hydrogen intake in carbon materials is in agreement with the knowledge that amorphous carbons are better absorbers than crystalline carbons [12].

Similar results are obtained in Figure 3 for the hydrogenated samples. Actually, the diffraction is a powerful tool to distinguish between irradiated and non-irradiated materials, but not very reliable to evaluate the level of over hydrogenation.

In the same way, if we proceed to evaluate the crystalline size $L_{c}$ using the Scherrer's formula [13], as shown in Table 3, the effect of the irradiation is to diminish $L_{c}$.

Similar evolution of XRD is known in carbon nanotubes irradiated with $\gamma$-rays [14].

The electrical properties of the samples have been obtained using the Van der Paw's technique described above and the results are shown in Table 4, where it is possible to see that the absorption of hydrogen decreases the electrical conductivity, as, it is known for similar materials [15].

The influence of the irradiation process is effective regarding the change in Seebeck's coefficient. In Table 4 we can observe that the irradiation per se improves the

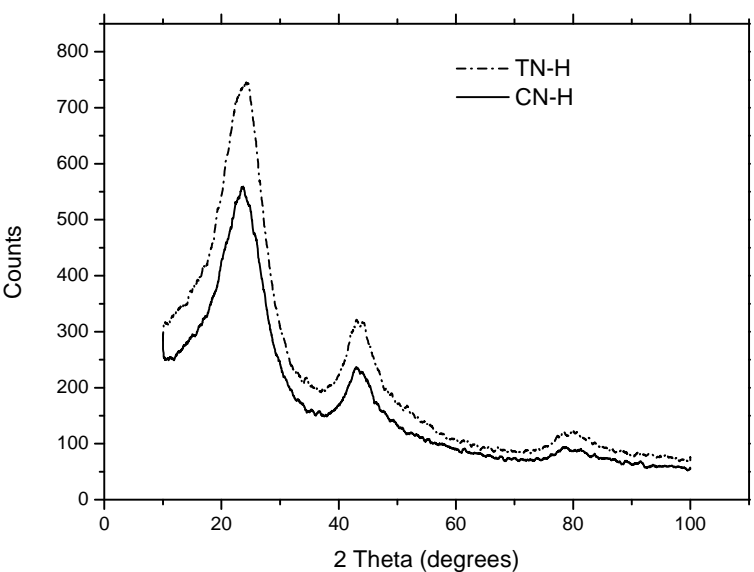

Figure 3. XRD plot of hydrogenated samples.

Table 3. Determination of crystalline size using the Scherrer's formula.

\begin{tabular}{cc}
\hline Sample & $L[\AA]$ \\
\hline CN-H & 6.8 \\
CN-sH & 7.3 \\
TN-H & 7.1 \\
TN-sH & 7.3 \\
\hline
\end{tabular}

Table 4. Results of the measurements of electric conductivity and Seebeck's effect of the carbon black samples.

\begin{tabular}{ccc}
\hline Sample & $\sigma(\mathrm{S} / \mathrm{cm})$ & $S(\mu \mathrm{V} / \mathrm{K})$ \\
\hline TN-sH & 2.00 & $0.87 \pm 0.01$ \\
TN-H & 1.49 & $4.2 \pm 0.2$ \\
CN-sH & 1.41 & $2.63 \pm 0.05$ \\
CN-H & 1.09 & $3.25 \pm 0.05$ \\
\hline
\end{tabular}

Seebeck's coefficient, but it is also remarkable that the intake of hydrogen increases that coefficient.

\section{Conclusion}

According to the above results, it is possible to conclude that the use of previous $\gamma$-irradiation improves the hydrogen intake at room temperature in carbon black from an over-pressurized atmosphere. The carbon black is converted into a more amorphous material by the effect of the irradiation. The X-ray diffraction is a valid technique to observe the changes that take place in the carbon black as a consequence of $\gamma$-irradiation and the alteration in the crystalline structure is explained by the change in the lattice parameter $L_{c}$.

\section{Acknowledgements}

We would like to acknowledge support from the Spanish 
Ministry of Finances and Competitivity (MINECO), through the grant of the Consolider-Ingenio 2010 project Nanotherm (CSD2010-00044).

\section{REFERENCES}

[1] S. Jaybhaye, M. Sharon, D. Sathiyamoorthy and K. Dasgupta, "Semiconducting Carbon Nanofibers and Hydrogen Storage," Synthesis and Reactivity in Inorganic, MetalOrganic, and Nano-Metal Chemistry, Vol. 37, No. 6, 2007, pp. 473-476.

http://dx.doi.org/10.1080/15533170701471729

[2] B. Panella, M. Hirscher and S. Roth, "Hydrogen Adsorption in Different Carbon Nanostructures," Carbon, Vol. 43, No. 10, 2005, pp. 2209-2214. http://dx.doi.org/10.1016/j.carbon.2005.03.037

[3] M. Molina-Sabio and F. Rodríguez-Reinoso, "Role of Chemical Activation in the Development of Carbon Porosity," Colloids and Surfaces A: Physicochemical and Engineering Aspects, Vol. 241, No. 1, 2004, pp. 15-25. http://dx.doi.org/10.1016/j.colsurfa.2004.04.007

[4] A. Rejifu, H. Noguchi, T. Ohba, H. Kanoh, F. RodriguezReinoso and K. Kaneko, "Adsorptivities of Extremely High Surface Area Activated Carbon Fibres for $\mathrm{CH}_{4}$ and $\mathrm{H}_{2}$," Adsorption Science and Technology, Vol. 27, No. 9, 2009, pp. 877-881. http://dx.doi.org/10.1260/0263-6174.27.9.877

[5] F. Banhart, "Irradiation Effects in Carbon Nanostructures," Report on Progress in Physics, Vol. 62, No. 8, 1999, pp. 1181-1221. http://dx.doi.org/10.1088/0034-4885/62/8/201

[6] V. Skakalova, U. Dettlaff-Weglikowska and S. Roth, "Gamma-Irradiated and Functionalized Single Wall Nanotubes," Diamond and Related Materials, Vol. 13, No. 2, 2004, pp. 296-298. http://dx.doi.org/10.1016/j.diamond.2003.11.003

[7] E. Papirer, S. Li, H. Balard and J. Jagiello, "Surface Energy and Adsorption Energy Distribution Measurements on Some Carbon Blacks," Carbon, Vol. 29, No. 8, 1991, pp. 1135-1143.

http://dx.doi.org/10.1016/0008-6223(91)90031-D
[8] A. Ansón, J. Jagiello, J. B. Parra, M. L. Sanjuán, Ana M. Benito, W. K. Maser and M. T. Martinez, "Porosity, Surface Area, Surface Energy, and Hydrogen Adsorption in Nanostructured Carbons," Journal of Physical Chemistry $B$, Vol. 108, No. 40, 2004, pp. 15820-15826. http://dx.doi.org/10.1021/jp047253p

[9] J. de Boor and V. Schmidt, "Complete Characterization of Thermoelectric Materials by a Combined Van der Pauw Approach," Advanced Materials, Vol. 22, No, 38 2010, pp. 4303-4307. http://dx.doi.org/10.1002/adma.201001654

[10] M. M. K. Salem, P. Braeuer, M. V. Szombathely, M. Heuchel, P. Harting, K. Quitzsch and M. Jaroniec, "Thermodynamics of High-Pressure Adsorption of Argon, Nitrogen, and Methane on Microporous Adsorbents," Langmuir, Vol. 14, No. 12, 1998, pp. 3376-3389. http://dx.doi.org/10.1021/la970119u

[11] P. Malbrunot, D. Vidal, J. Vermesse, R. Chahine and T. K. Bose, "Adsorption Measurements of Argon, Neon, Krypton, Nitrogen and Methane on Activated Carbon Up to 650 MPa," Langmuir, Vol. 8, No. 2, 1992, pp. 577-580. http://dx.doi.org/10.1021/la00038a044

[12] V. Jiménez, A. Ramírez-Lucas, P. Sánchez, J. L. Valverde and A. Romero, "Hydrogen Storage in Different Carbon Materials: Influence of the Porosity Development by Chemical Activation," Applied Surface Science, Vol. 258, No. 7, 2012, pp. 2498-2509. http://dx.doi.org/10.1016/j.apsusc.2011.10.080

[13] J. I. Langford and A. J. C. Wilson, "Scherresr after Sixty Years: An Survey and Some New Results in the Determination of Crystalline Size," Journal of Applied Cristallography, Vol. 11, No. 2, 1978, pp. 102-113. http://dx.doi.org/10.1107/S0021889878012844

[14] Z. Xu, L. Chen, L. Liu, X. Wu and L. Chen, "Structural Changes in Multi-Walled Carbon Nanotubes Caused by $\gamma$-Ray Irradiation," Carbon, Vol. 49, No. 1, 2011, pp. 350-351. http://dx.doi.org/10.1016/j.carbon.2010.09.023

[15] C. Marliere, P. Poncharal, L. Vaccarini and A. Zahab, "Effect of Gas Adsorption on the Electrical Properties of Single Walled Carbon Nanotubes Mats," International Materials Reviews, Vol. 593, 2000, pp. 173-176. 